\title{
OPP Approach for Multi Degree of Freedom Robotic Arm Based on Kinematics and Dynamics of Robot
}

\author{
Rashmiranjan Das*, Rupali Mathur*, Deepika Rani Sona**, Praneet Dutta** \\ * School of Electrical Engineering, VIT University, Tamil Nadu, India \\ ** School of Electronic Engineering, VIT University, Tamil Nadu, India
}

\begin{tabular}{l} 
Article Info \\
\hline Article history: \\
Received Jul 10, 2015 \\
Revised Oct 25, 2015 \\
Accepted Nov 14, 2015 \\
\hline Keyword: \\
OPP \\
Pontrygain's principle \\
Cost function \\
kinodynamic constrains \\
DOF
\end{tabular}

\begin{abstract}
This paper deals with the trajectory and path generation of the industrial manipulator. The trajectory is obtained using the equations of motion and also the optimal path planning (OPP) approach under kinodynamic constraints. The optimal control problem is defined for the minimum cost function and to obtain the necessary conditions. Here we have used pontrygain's minimum principle to obtain the limiting value of joint angle and also the joint velocity and torque. In this paper we have used the "Two degree of freedom (DOF) manipulator" for analysis and designing the optimal control for multi link and multi degree of freedom manipulator. For analysis purposes, simulation software has been used to formulate the trajectory and minimize the cost function involved.
\end{abstract}

Copyright $(0) 2015$ Institute of Advanced Engineering and Science. All rights reserved.

\section{Corresponding Author:}

Rupali Mathur

School of Electrical Engineering

VIT University

Vellore 632014, Tamil Nadu, India

rupalimathur8@gmail.com

Deepika Rani Sona

School of Electronics

Engineering

VIT University

Vellore 632014, Tamil Nadu, India

deepika.rs@vit.ac.in

\section{INTRODUCTION}

In a plethora of industries, Robotics is an integral part of automation. The industrial robotic arm is used for various tasks right from the assembly, spray painting and welding, carrying parts from one place to another, packaging and many other tasks. For establishing a manipulator it is required to establish the trajectory path.

Without any constraints and the path the industry cannotgo ahead with the production process efficiently. To optimize our solution, we analyze the constraints on the joint angle, joint velocity and torque.

If the robotic arm were to move in a free trajectory it is capable of destroying large proportions of the workspace. As shown in fig-An automotive industry in which car is assembled using the robotic arm. Each and every robotic manipulator has its own trajectory defined. If the path has not been decided ,then the trajectory of the robot is lost and the damage has been inflicted by the manipulator which is dangerous for the industrial environment and people working in the industry. Here we use the kinodynamic constraints to formulate the trajectory. Kino dynamics refers to kinematics and dynamic formulation. Kinematics of the robot involves the formulation of $\mathrm{D}-\mathrm{H}$ parameter along with the jacobian matrix. The jacobian matrix 
involves the rotational and translation of the robotic arm. Trajectory generation involve two method The Former is the decoupled approach in which a feasible path is first formed and then optimization of control along the path takes place.The Second method involves a direct trajectory planning approach-It involves the kinodynamic constraints of the system to solve the optimal path planning approach. Direct trajectory planning approach is done by using the equations of motion. In this approach, the optimal solution is derived from the state space of the system and it solves an unknown optimal trajectory of each joint.In this Optimal control problem, we will first solve the two degree of freedom robotic manipulator. Hence, using the constraints we will define the trajectory of the robotic arm.

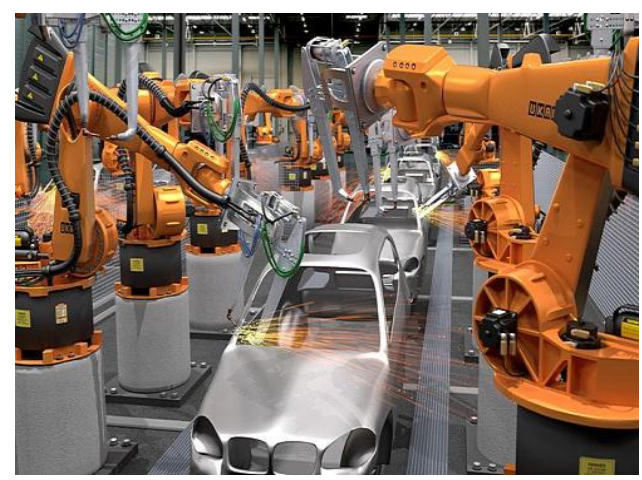

Figure 1. Automotive industry

\section{BLOCK DIAGRAM}

The block diagram used to calculate the trajectory of the path is:

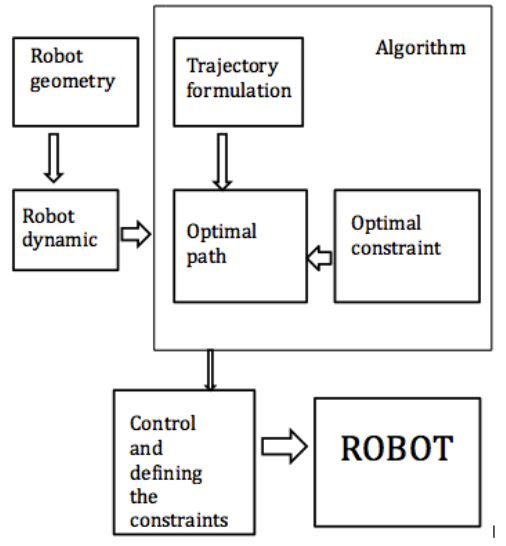

Figure 2. Block diagram

According to this, initially the robot geometry is defined along with robot dynamics such as velocity, kinetic and potential energy of the robotic joint. Then we formulate the optimal control algorithm. Using the trajectory equation, the following are also set- the optimal constraints on the joint angle, joint velocity, force and torque. These constraints are applicable on the optimal path. This trajectory is defined in the control of the robotic manipulator. Equations of motion are applied to plan the trajectory and pontrygain principle to obtain the optimal value of the joint angle velocity and acceleration.

\section{EQUATIONS AND CALCULATIONS}

Equation of Motion:

$\mathrm{D}(\mathrm{q}) \ddot{q}+\mathrm{C}(\mathrm{q}, q) \dot{q}+\mathrm{g}(\mathrm{q})=\mathrm{Q}$ 
Where $\mathrm{q}$ is the vector of generalized coordinates $\left(\theta_{1}, \theta_{2}\right.$ in this case), $\mathrm{D}$ is a positive definite inertia matrix, $\mathrm{C}$ is the velocity coupling vector, $\mathrm{g}$ is the gravitation force vector, and $\mathrm{Q}$ is the generalized force vector or the joint torques $\tau_{1} \tau_{2}$ in this case.

Performance measure is given by $\mathrm{J}=0.5^{*} \mathrm{x}{ }^{\prime} \mathrm{Px}+0.5 \int\left(x^{\prime} Q x+u^{\prime} R u\right) d x$ where $\mathrm{P}$ and $\mathrm{Q}$ are positive semi definite matrix and $\mathrm{R}$ is positive definite matrix. $\mathrm{X}$ is the state vector and $\mathrm{U}$ is the control vector. For optimal control problem we follow three steps to obtain the optimal value of joint angle $\theta$. The first involves the problem formulation which is defined by the kinematics of the robot arm .Initially we take two degree of freedom for the robotics arm to formulate the equations. The second step consists of defining the constraints applying physical constraints to the robotic arm. Third is the formulation of the performance measures to obtain the optimal value of joint angle. Consider the two degree of freedom robotic arm as shown in figure.

Defining the kinematic parameter $a_{1}$ and $a_{2}$ are the link length $d_{1}$ and $d_{2}$ are the link offsets, $\theta_{1}$ and $\theta_{2}$ are joint angle. $\mathrm{x}, \mathrm{y}, \mathrm{z}$ define the rotational axis. 2-DOF link manipulator has the end affecter of different shapes it can be a welding torch or a paint brush, clamper etc.

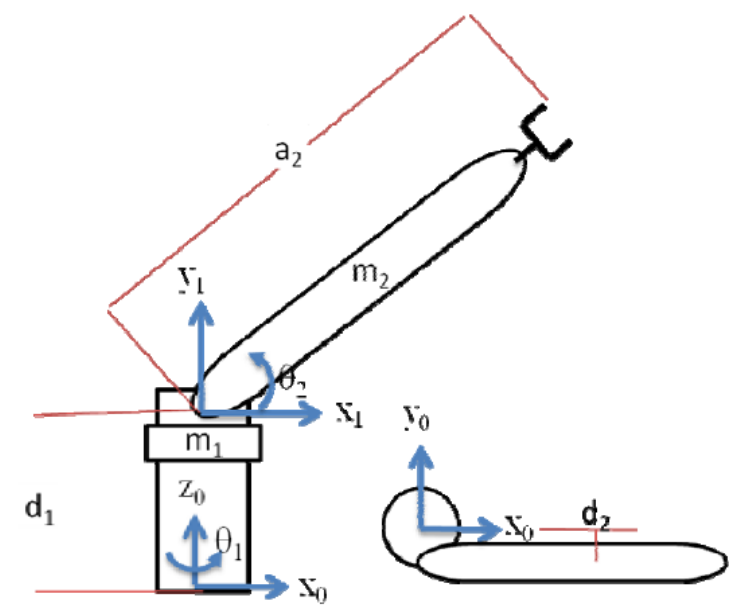

Figure 3. Two degree of freedom robotic arm

Apply the Denavit-Hartenberg homo-geneous transfer matrix used to characterize the kinematics of robotic arm. Using rotations and translational

$$
\begin{aligned}
& {\left[\begin{array}{l}
x \\
y \\
z
\end{array}\right]=\left[\begin{array}{ccc}
\cos \theta_{1} & -\sin \theta_{1} & 0 \\
\sin \theta_{1} & \cos \theta_{1} & 0 \\
0 & 0 & 1
\end{array}\right]\left(\begin{array}{l}
1 \\
1 \\
1
\end{array}\left[\begin{array}{c}
a_{1} \\
-d_{2} \\
d_{1}
\end{array}\right]+\left[\begin{array}{ccc}
\cos \theta_{2} & 0 & -\sin \theta_{2} \\
0 & 1 & 0 \\
\sin \theta_{2} & 0 & \cos \theta_{2}
\end{array}\right]\left[\begin{array}{l}
a_{2} \\
0 \\
0
\end{array}\right]\right)} \\
& \mathrm{x}=\mathrm{a}_{1} \cos \theta_{1}+\mathrm{d}_{2} \sin \theta_{1}+\mathrm{a}_{2} \cos \theta_{1} \cos \theta_{2} \\
& \mathrm{y}=\mathrm{a}_{1} \sin \theta_{1}-\mathrm{d}_{2} \cos \theta_{2}+\mathrm{a}_{2} \sin \theta_{1} \cos \theta_{2} \\
& \mathrm{z}=\mathrm{d}_{1}+\mathrm{a}_{2} \sin \theta_{2}
\end{aligned}
$$

These equations are used for calculating the values of the constraints and performance measures. Applying the two degree of freedom dimensions and limits as for link 1 that is $\mathrm{a}_{1}=0.0 \mathrm{~m}, \mathrm{~d}_{1}=10 \mathrm{~cm}$, for link2 $\mathrm{a}_{2}=48 \mathrm{~cm}, \mathrm{~d}_{2}=2 \mathrm{~cm}$. By calculation we obtain the value of torque, angular velocity and acceleration. As for link1 it is $\tau_{1}=2.4 \mathrm{Nm}, \tau_{2}=2.9 \mathrm{Nm}, \omega_{1}=7.5 \mathrm{rad} / \mathrm{sec}, \omega_{2}=7.5 \mathrm{rad} / \mathrm{sec}, \alpha_{1}=7.5 \mathrm{rad} / \mathrm{sec}^{2} \alpha_{2}=10 \mathrm{rad} / \mathrm{sec}^{2}$.

Forming the constraint equations by applying the following conditions on state vector and control vector as: 
$\mathrm{X}=\left[\begin{array}{l}\theta_{1} \\ \dot{\theta_{1}} \\ \theta_{2} \\ \dot{\theta_{2}}\end{array}\right] \varepsilon \mathrm{X}:=\left\{\begin{array}{c}\theta_{1}:-90 \leq \theta_{1} \leq 90 \\ \theta_{2}: 45 \leq \theta_{2} \leq 135 \\ \dot{\theta}_{1}:-7.5 \leq \dot{\theta}_{1} \leq 7.5 \\ \dot{\theta}_{2}:-7.5 \leq \dot{\theta}_{2} \leq 7.5\end{array}\right\}$

Control vector $\mathrm{u}=\left[\tau_{1} \tau_{2}\right]^{\mathrm{T}} \varepsilon \mathrm{U}:=\left\{\begin{array}{l}\tau_{1}:-2.4 \leq \tau_{1} \leq 2.4 \\ \tau_{2}:-2.9 \leq \tau_{2} \leq 2.9\end{array}\right\}$

Minimize $\mathrm{J}\left[\mathrm{x}(), \mathrm{u}(), \mathrm{t}_{\mathrm{f}}\right]=\mathrm{t}_{\mathrm{f}}$

Subject to: $\dot{x}(\mathrm{t})=\left[\begin{array}{c}\theta_{1} \\ \dot{\theta_{1}} \\ \theta_{2} \\ \dot{\theta_{2}}\end{array}\right]=\left[\begin{array}{c}\omega_{1} \\ \omega_{2} \\ \alpha_{1} \\ \alpha_{2}\end{array}\right]$ where $\alpha_{\mathrm{i}}=\mathrm{f}(\mathrm{x}, \mathrm{u})$

$\mathrm{x}\left(\mathrm{t}_{0}\right)=\left[\begin{array}{c}\theta_{1}^{0} \\ \theta_{2}^{0} \\ 0 \\ 0\end{array}\right]$

$\mathrm{e}\left(\mathrm{x}_{\mathrm{f}}, \mathrm{t}_{\mathrm{f}}\right)=\left[\begin{array}{c}a_{1} \cos \theta_{1}+d_{2} \sin \theta_{1}+a_{2} \cos \theta_{1} \cos \theta_{2}-x^{f} \\ a_{1} \sin \theta_{1}-d_{2} \cos \theta_{2}+a_{2} \sin \theta_{1} \cos \theta_{2}-y^{f} \\ d_{1}+a_{2} \sin \theta_{2}-z^{f} \\ \omega_{1}^{f} \\ \omega_{2}^{f}\end{array}\right]=[0]$

$\left[\begin{array}{l}x^{L} \\ u^{L}\end{array}\right] \leq h(x, u) \leq\left[\begin{array}{l}x^{u} \\ u^{u}\end{array}\right]$

Now applying the necessary conditions-To be optimal ,the solution must satisfy the pontrygain's principle. Define the Hamiltonian, $\mathrm{H}$ as a function of running cost, $\mathrm{F}$, the vector of co-states $\lambda(t)$, and the principle as:

$$
\mathrm{H}(\lambda, \mathrm{x}, \mathrm{u}, \mathrm{t}):=\mathrm{F}+\lambda^{\mathrm{T}} \mathrm{f}
$$

\subsection{SCENARIO 1}

Now applying the given above configurations and calculating it with the simulation and obtaining the results applying the $1^{\text {st }}$ scenario as given:

$\theta_{1}^{0}=0, \theta_{1}^{f}=90, \theta_{2}^{0}=45, \theta_{2}^{f}=45$ the result for this is as

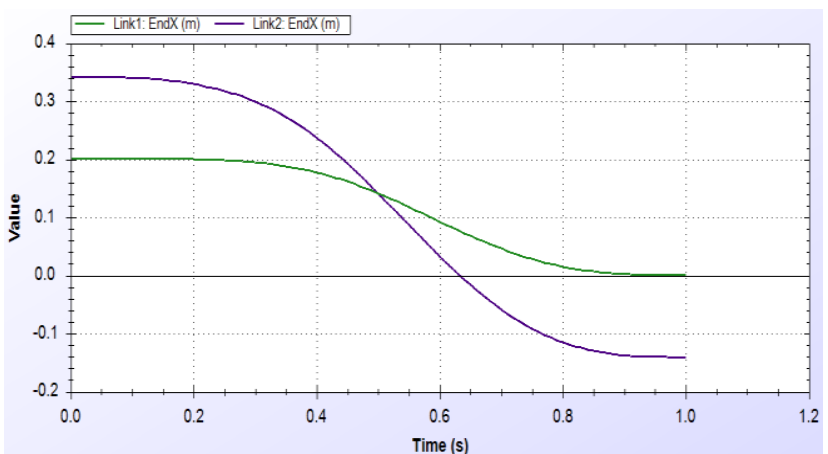

Figure 4a. Link1 parameter of End X

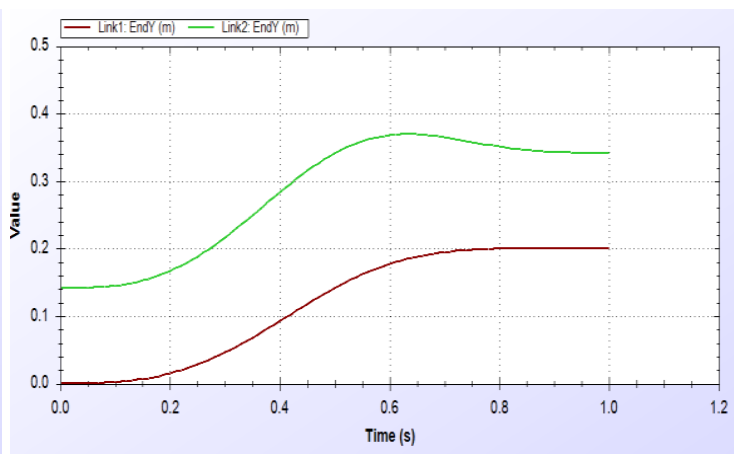

Figure $4 \mathrm{~b}$. Link $1 \&$ link 2 parameter end y 


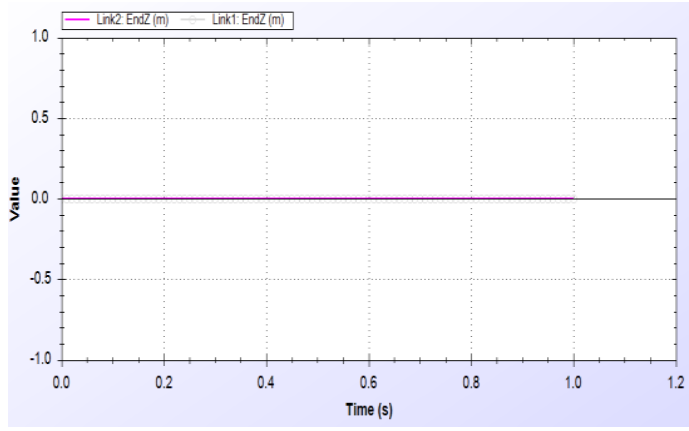

Figure 4c. Link1\& link2 parameter end z

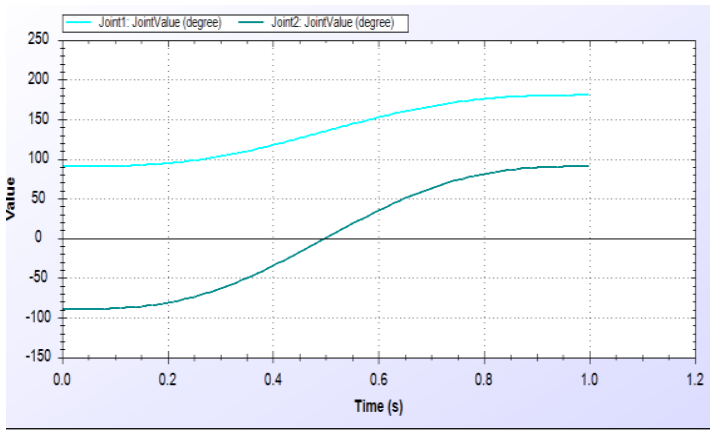

Figure 5. Joint value of scenario 1

In the joint velocity plot for link $1 \&$ link 2 the cycloid trajectory is represented as in the shape of cycloid.From the figure we can see that the shape of the curve is varying at different values of joint velocity. Joint acceleration with the inverse dynamic constraints gives this plot.

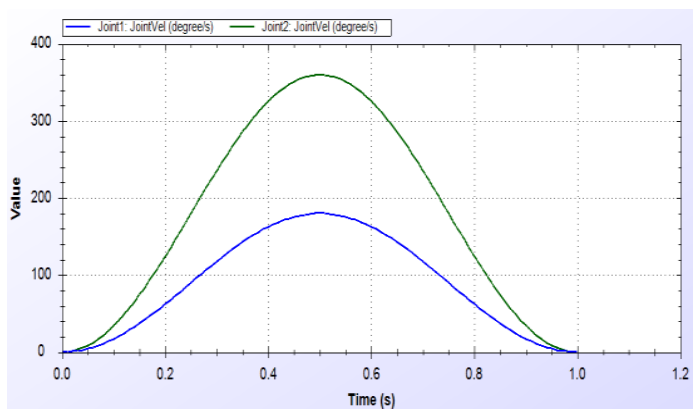

Figure 5a. Joint velocity of link1 and link 2

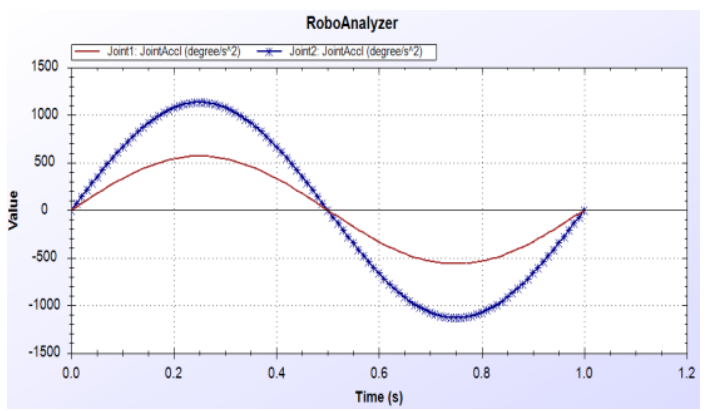

Figure 6. Joint acceleration of link $1 \&$ link 2

Force and torque with the inverse kinematic constraints.

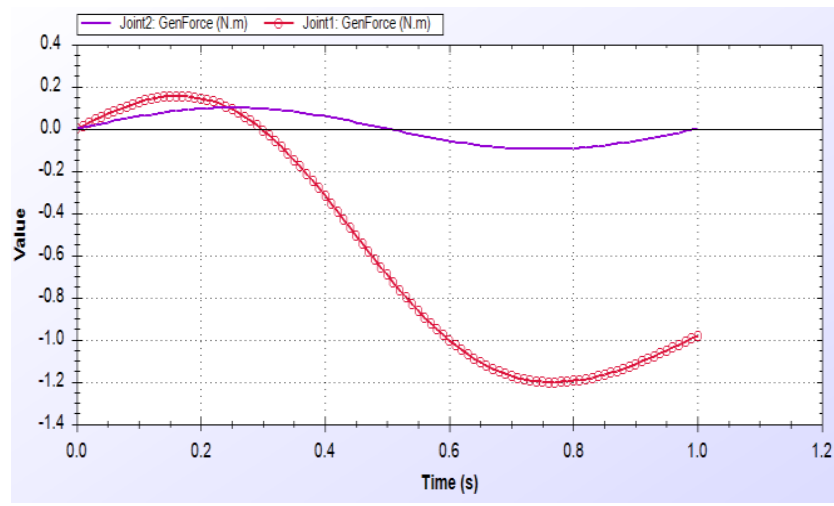

Figure 7. Force/torque of the joint $1 \&$ joint 2

\subsection{SCENARIO 2}

Now calculating the constraints value in the second scenario

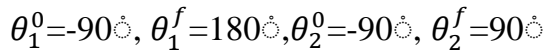


The value of joint angle and joint velocity will vary in this scenario because the trajectory it forms will be a circular one starting from one end and ending at the other.

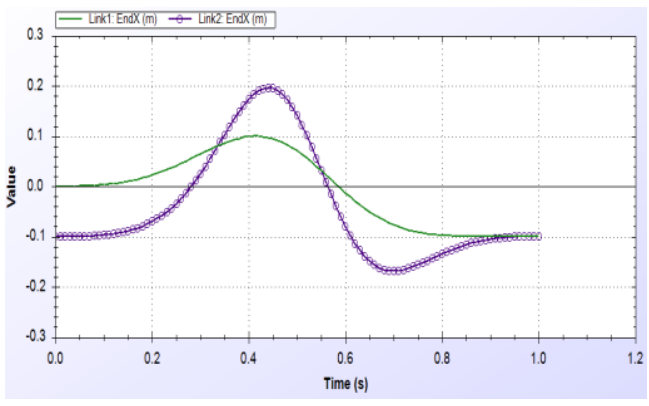

Figure 8a. Link $1 \&$ link $2 \mathrm{x}$-axis plot

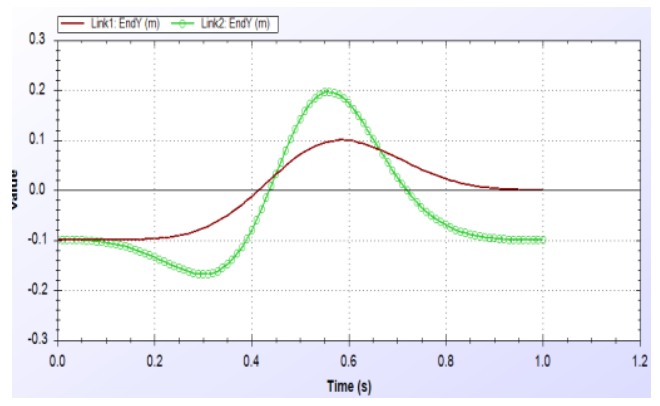

Figure $8 b$. Y axis plot

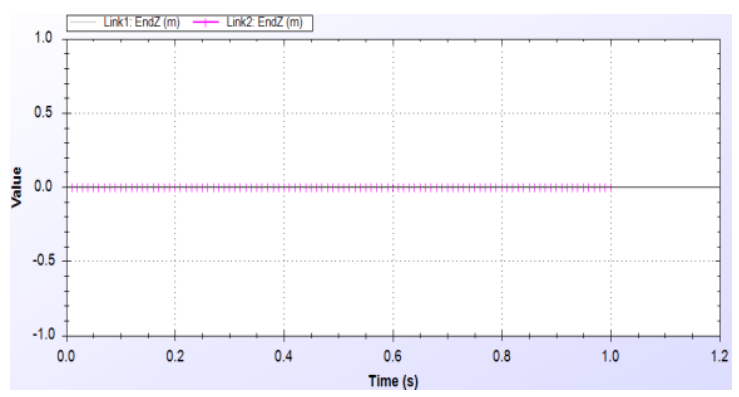

Figure 8c. $\mathrm{Z}$ axis plot

The joint angle plot, joint velocity plot, and joint acceleration plot is given as for both the link $1 \&$ link 2:

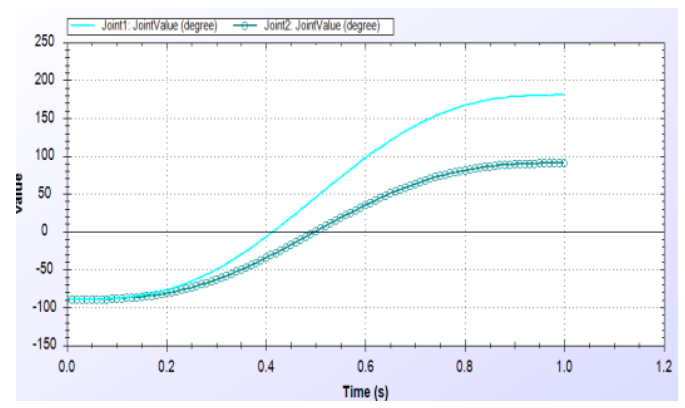

Figure 9. Joint angle plot of scenario 2

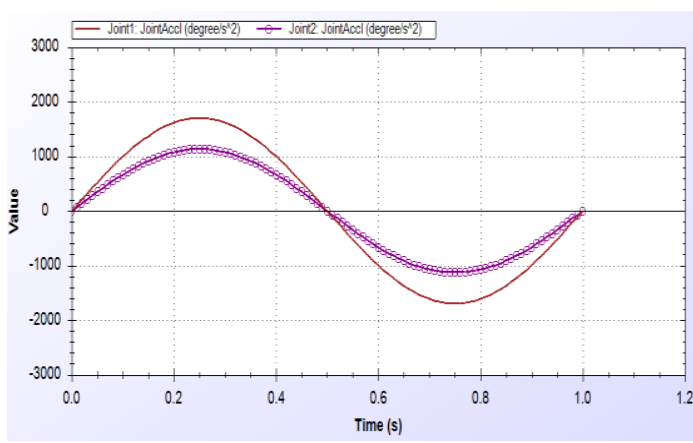

Figure 11. Joint acceleration of scenario 2

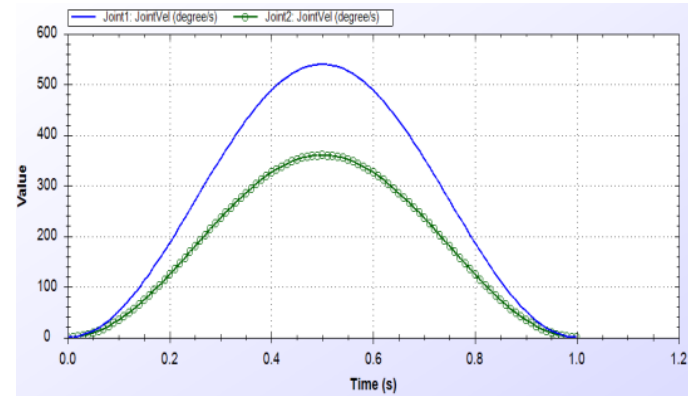

Figure 10. Joint velocity of scenario 2

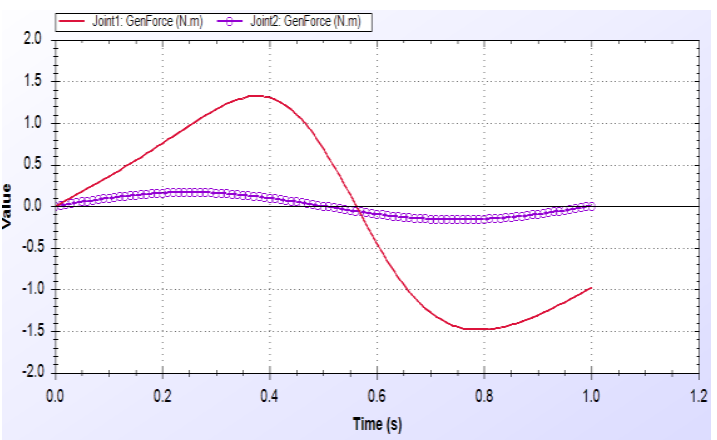

Figure 12. Force \& torque of scenario 2 


\section{RESULT}

We have compared the two scenarios and have obtained the following results. In the first scenario the trajectory is generated as a quarter circle and in the second scenario the trajectory is generated as a full circle starting ending at the same point.

Taing the mean of the link values and consolidating it into a tabular form we get:

Table1. Comparison between the values of scenario1 \& scenario2

\begin{tabular}{lll}
\hline Characteristic & Scenario1 & Scenario2 \\
\hline Link X- axis & $0.54 \mathrm{~m}$ & $0.50 \mathrm{~m}$ \\
Link y- axis & $0.29 \mathrm{~m}$ & $0.15 \mathrm{~m}$ \\
Link Z-axis & $0.0022 \mathrm{~m}$ & $0.0012 \mathrm{~m}$ \\
Joint angle & $45 \mathrm{deg}$ & $50 \mathrm{deg}$ \\
Joint velocity & $90 \mathrm{deg} / \mathrm{sec}$ & $100 \mathrm{deg} / \mathrm{sec}^{2}$ \\
Joint acceleration & $340 \mathrm{deg} / \mathrm{sec}^{2}$ & $300 \mathrm{deg} / \mathrm{sec}^{2}$ \\
Force\& torque & $0.1757 \mathrm{Nm}$ & $0.0995 \mathrm{Nm}$ \\
\hline
\end{tabular}

As the trajectory generated is also different in these cases-One is quarter shaped and the other is circular.

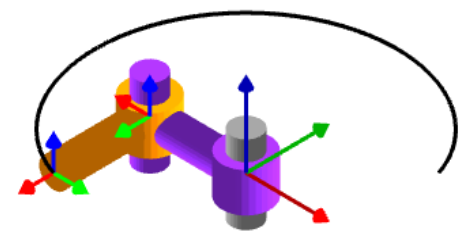

Figure 13. Scenario 1 output

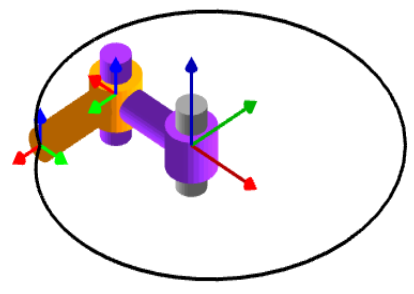

Figure 14. Scenario 2 output

By studying the figure, one can observe the difference in the trajectory formation as displayed in Figure 13.It is beneficial for picking up and placement of objects. Figure 14 displays a trajectory which is more suited for welding.

\section{COMPARISON}

Table2. Comparison between 2 DOF and multi link manipulator

\begin{tabular}{lll}
\hline Characteristic & $2 \mathrm{DOF}$ & SCARA \\
\hline Link length & $0.284 \mathrm{~m}$ & $0.546 \mathrm{~m}$ \\
Joint value & $47.5 \mathrm{deg}$ & $58 \mathrm{deg}$ \\
Joint velocity & $95 \mathrm{deg} / \mathrm{sec}$ & $105 \mathrm{deg} / \mathrm{sec}$ \\
Joint acceleration & $150 \mathrm{deg} / \mathrm{sec}^{2}$ & $165 \mathrm{deg} / \mathrm{sec}^{2}$ \\
Force/ Torque & $0.1787 \mathrm{Nm}$ & $0.602 \mathrm{Nm}$ \\
\hline
\end{tabular}

\section{FUTURE SCOPE}

This methodology can be used for generating the optimal path for a unmanned autonomous vehicle or a aircraft controlling in the military applications.

\section{REFERENCES}

[1] A General, Fast, and Robust Implementation of the Time-Optimal Path Parameterization Algorithm Quang-Cuong Pham. IEEE transaction on robotics, vol.30, no.6, December 2014.

[2] Optimal path planning for multi arm, multilink robotic manipulator by Joseph A. Cascio December 2008. 
[3] A cooperative path planning algorithm for multiple mobile robot system in a dynamic environment regular. Wentao Yu1, Jun Peng1,*, Xiaoyong Zhang1 and Kuo-chi Lin2. International Journal of Advanced Robotic Systems, June 2014.

[4] Reactive Path Planning in a Dynamic Environment Fethi Belkhouche, member IEEE transaction on robotics, vol.25, no.4,august 2009.

[5] Vision based, distributed control laws for motion cordination of nonholonomic robots, Nima Moshtagh, member IEEE, Nanthan Michael, member IEEE, Ali jadbabaie, senior member IEEE and Kostas Daniilids IEEE transcation on robotics, vol.25,no.4, august 2009.

[6] Time Optimal Motion Planning and Motion Control for Industrial Manipulators. Norwegian university of Science \& Technology. May 2014(master thesis)

[7] Figure1- from the automotive industry working environment. 